\title{
Namenregister zu Band 58
}

Die fettgedruckten Zahlen bedeuten Eigenarbeiten.

Zeitschrift für àugenheükundö. Bd. 58. Heft 6.

A.

d'Amio Diego 251. Arnold 390. Ascher 237. Asmus 187. Aszalós 383. Aust 374, 450. Avizonis 343. Axenfeld 244.

B.

Bacl·ïem 459. Back 232. Backhaus 224. Becker 240. Behr 27, 224, 398, 458. Bélés 248. Berger 227. Bielschowsky 57, 216. Bittorf 227. Blaauw 186. v. Blaskovics 383, 384. Blatt 217.

Bliedung 190 (P.). Böhm 230. Bolesch 240. Braun 235. Brieger 407. Brouwer 225. Bruckner 74.

C. Carsten 210. Cedercreutz 186. Comberg 92, 171, 314

(P.). Conway 385. Cramer 460. v. Csapody 248, 382. Curschmann 221, 245.

D.

Derby 312.

Dimmer 392 (P.), 393

(P.) Dohme 38. Duke-Elder 308. Dupuy-Dutemps 387.

E.

Eigel 244. Eiselsberg 223. Eisendraht 220. Elschnig23i, 232, 236, 237, 461.

F.

v. Fazakas 382. Feingold 392 (P.). v. Ferenczy 383. Feuchtwanger 226. Fischer-Ascher 458.

Fleischer 181, 375. v. Fodor 382. Freudenberg 390. Fuchs 315. Fuss 419.

G.

Gerson 310. Gleichen 250. Goldstein 227. Gradle 220. Grosser 230.

H.

Hamann 167. Hamburger 185. Hanke 444. Heesch 391. Heine 191, 194, 222. Hemmer 413. v. Herrenschwand

222. Herrmann 227-Herrmannsdorfer

310. van Heuven 238, 239. Heymann 390. Hildesheimer 233. vom Hofe 388. Hofer 180.

Hoffmann, W. 465 (P.) Hofmann, F. B. 42. Hönig 236. Horay 249. Horváth 384. Houwer 239.

Huber 382, 383, 384. Hübner 358.

I.

v. Imre sen. 383 .

J.

Jablonski 246. Jaensch 2. Jamin 457. Jüngst 459.

K.

Kafka 375.

Karpow 219, 220. Kauffmann 221, 222. Kaufmann 241. Kehrer 219. Kestenbaum 179, 377.

Kestermann 216. Kleefeld 311. Klein 250. Köhler, G. D. 464. Königsfeld 389. Kranz 390.

Kreiker 190 (P.), 382, 
383. Kreutzfeld 222. Kronfeld 180, 216, 447,

449, 45 . Krückmann 184. Kubik 223, 234, 236. Kuffler 115. Kühl 175. Kyrieleis 202.

L.

Lang 377. Lechler 217. Lehmann 241. Leitner 248. Levinsohn 217. Licskó 249. v. Liebermann

247. Lindner 183, 307, 308,

452, 453-Lochtkemper 461. Löwenstein 230, 232,

238.

M.

Maas 224. Marburg 253. Marches ani 188. Marschik 178. Matzdorff 217. Meesmann 184, 384.

Meisner 128. Meiß ner 178. Meller 180, 377. Memmesheimer 220. Metzger 222. Meyer, A. 219, 220. Mylius 295.

N. Najditsch 299. Nelisssen 220. Niehues 460.

31

$474 \quad$ Namenregister zu Bd. 58.

0 .

Ohm 445. Ovio 313.

P.

Paderstein 184. Paunz 248. Payr 189. Périn 387. Peters 17, 188. Pillat 275, 308, 426, 443-Plog 222. Pometta 463. Pötzl 233. Pribram 232. Procksch 444.

R.

Rabitsch 206. Reckzeh 461. Reinhardt 222. Reitsch 246. Rieger 370. Rochat 238, 239. Roelofs 238. Rohrschneider 12,

184. Rosenberg, A. 138. Rosenstein, A. M. 189. Rotter 234.

S. Sachs 303, 307. Safar 180, 269, 378, 443. Sallmann307, 348,369, 372, 374-

Salus 218, 233, 237,

238. Salzer 392. Samojloff 214, 282. Sauerbruch 310. Schacherl 225. Schlippe 217. Schneider

115. Schön 220. Schridde 459. Schubert 175. Schulze, H. 250. Schwartz 216. v. Seggern 225.

Segi 226. Seidler 307. Seißiger 241. Sittig 227. Sommer 182, 376. Somogyi 250. Stanka 237.

Stargardt 391. Steindorff 462. Stock 245. Stoewer 92. Strebel 464. Stübel 242. Sypkens 239.

Székács 250.

T.

Takagi 436. Tapasztó 383. Taußig 375. Teleky 389. Thiel 86, 190 (P.).

Thies 242. van Thye 239 .

U.

Uhthoff 225. Uudelt 288.

V.

Velhagen 242. Verderber 292. Vogt 379, 380, 381.

W. Waetzold 139. Waldmann 248, 249. Weber, F. 368. Wegner 123. Weichbrodt 462. Weintraub 306. * Weizenblatt 454. Werdenberg 455. Weve 239. Wibaut 220, 239. Wick 216. Wieland 220. Wilbrand 197, 261. Wildi 243. Wirth 106. Wißmann 190 (P.), 250.

Z.

Zeiss ..243. Ziemssen 165. Zirm 231, 237. Zollinger 463. Zweifel 189. 